\title{
Glasses for 3D Ultrasound Computer Tomography
}

\section{Background}

At KIT at Karlsruhe we are developing a new imaging
method for early breast cancer detection: 3D ultrasound computer tomography [1] The prototype currently in clinical study trial is called 3DUSCT II. The basic concept is full 3D synthetic aperture focussing (SAFT) ${ }^{[2]}$ with unfocussed spherical waves emitted and received iteratively by individual transducers. The measurement happens in a semi-ellipsoidal formed water-filled container $(17 \mathrm{~cm} \times 24 \mathrm{~cm}$, height and width $)^{[3]}$ which can be lifted and rotated. The walls are lined with thousands of transducers forming the imaging aperture. The used ultrasound transducers have a resonance frequency at $2.6 \mathrm{MHz}, 50 \%$ relative bandwidth Figure 1: 3DUSCT II (2013)

\section{and a directivity of $\pm 23^{\circ}$ at $-6 \mathrm{~dB}$. \\ Motivation and Idea}

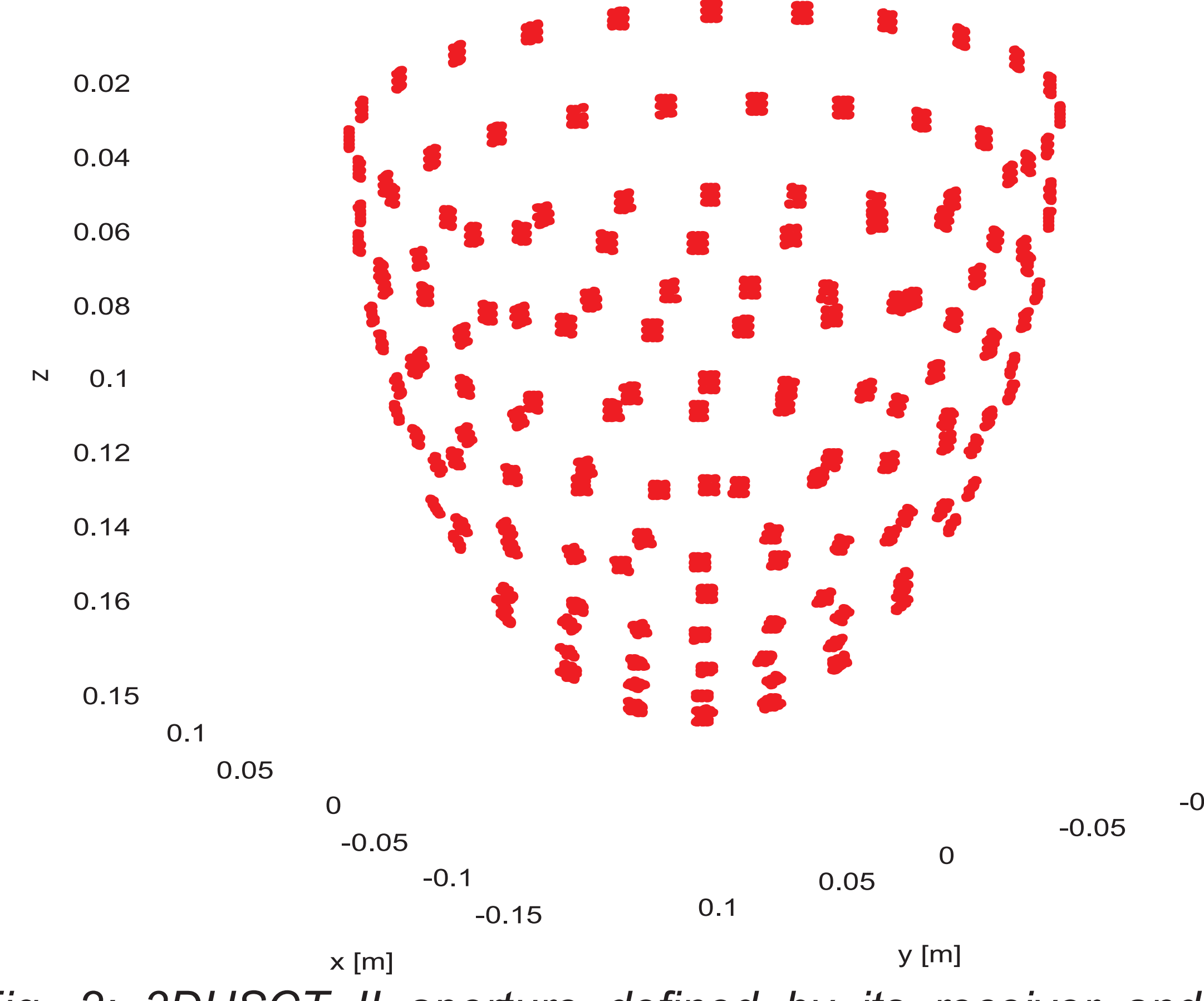

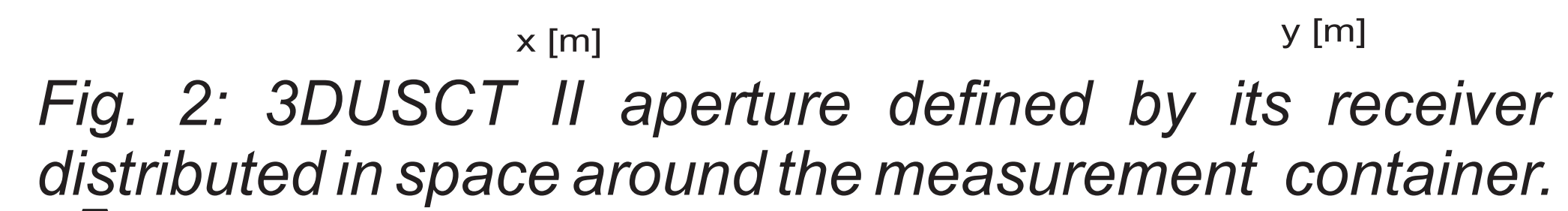

Imaging can be described in the spatial Fourie domain ( $\mathrm{k}$-space) as linear process ${ }^{44}$, i.e. as multiplication of the object function with the imaging function given by the aperture. SAFT apertures with significant spatial extend in relation to the object size, especially object enclosing apertures, exaggerate lowfrequency components ${ }^{2}$. These overamplified object frequencies are the reason for a blurred characteristic in straight-forward SAFT imaging ${ }^{[5]}$.

\section{Aperture representation in k-space}

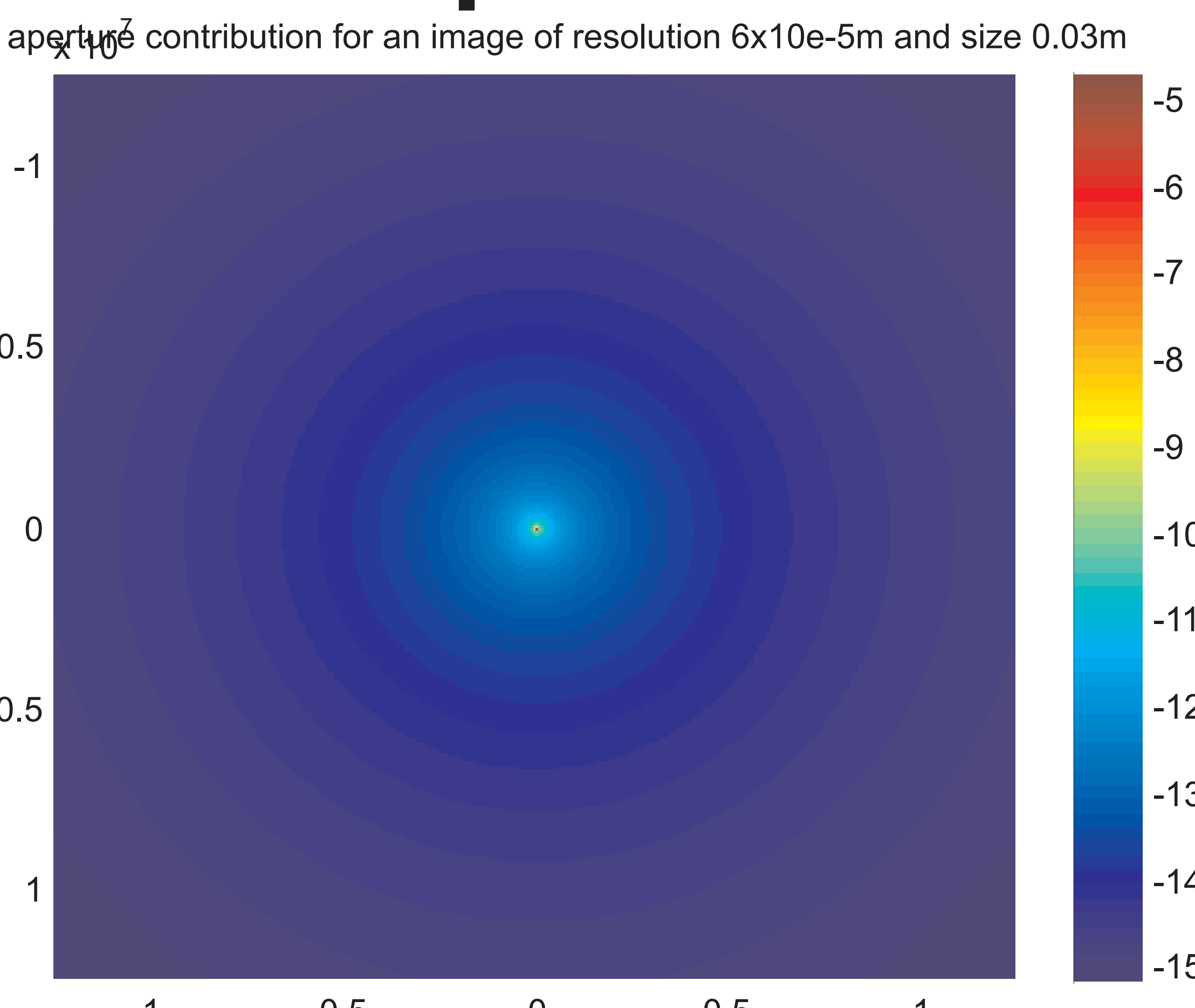

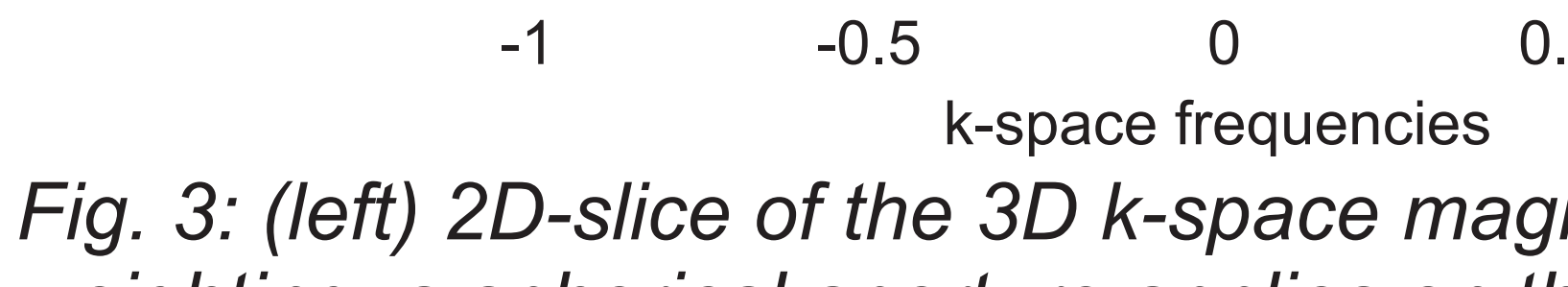

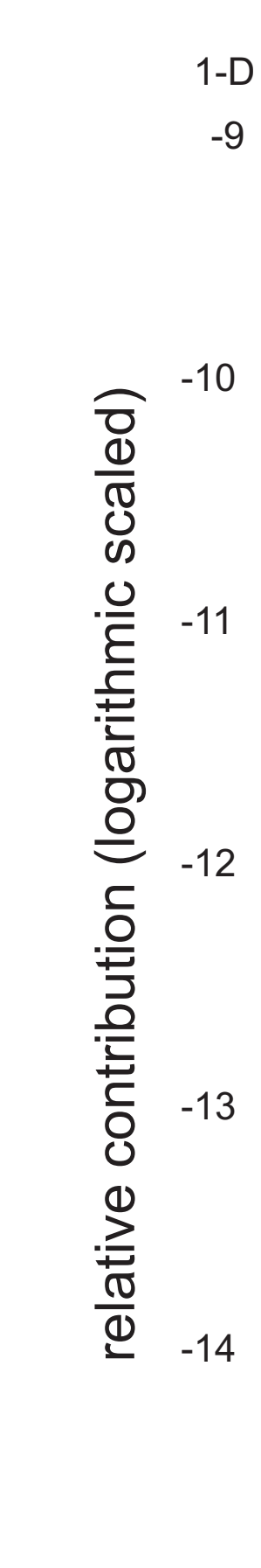

M. Zapf, N.V. Ruiter

\section{Methods}

The 3DUSCT II aperture was modeled by its spatial position of the ransducer elements and the frequency characteristic of the ultrasound ransducers (center frequency $2.6 \mathrm{MHz}$ and $50 \%$ relative bandwidth). As simulated objects perfect point scatterers were chosen as they have a known uniform k-space contribution. A resolution metric was extracted by applying a contour filter and defining the mean full-width-halfmaximum (FWHM) as resolution per point scatterer.

The aperture compensation filter the 'glasses', was applied to the constructed images, cor the image blur introduced by the aperture and imaging process and enhancing the resolution.
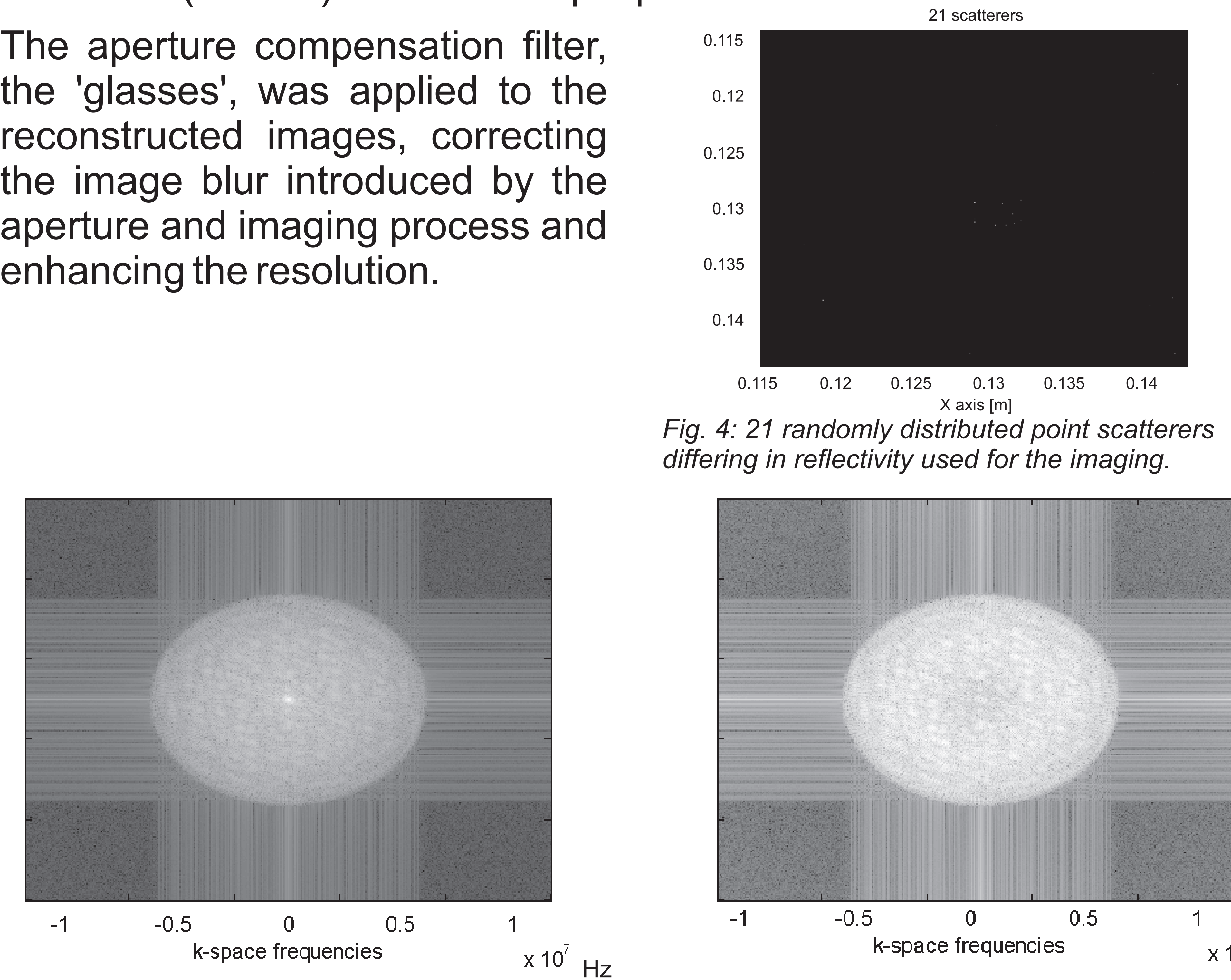

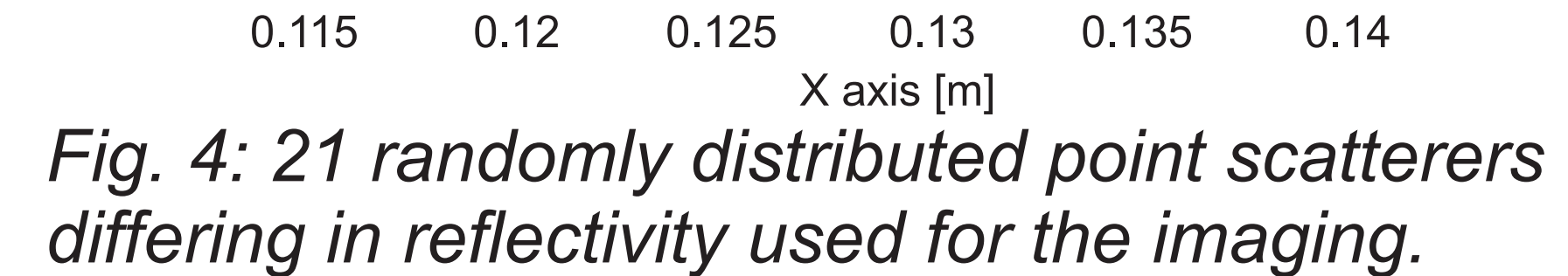
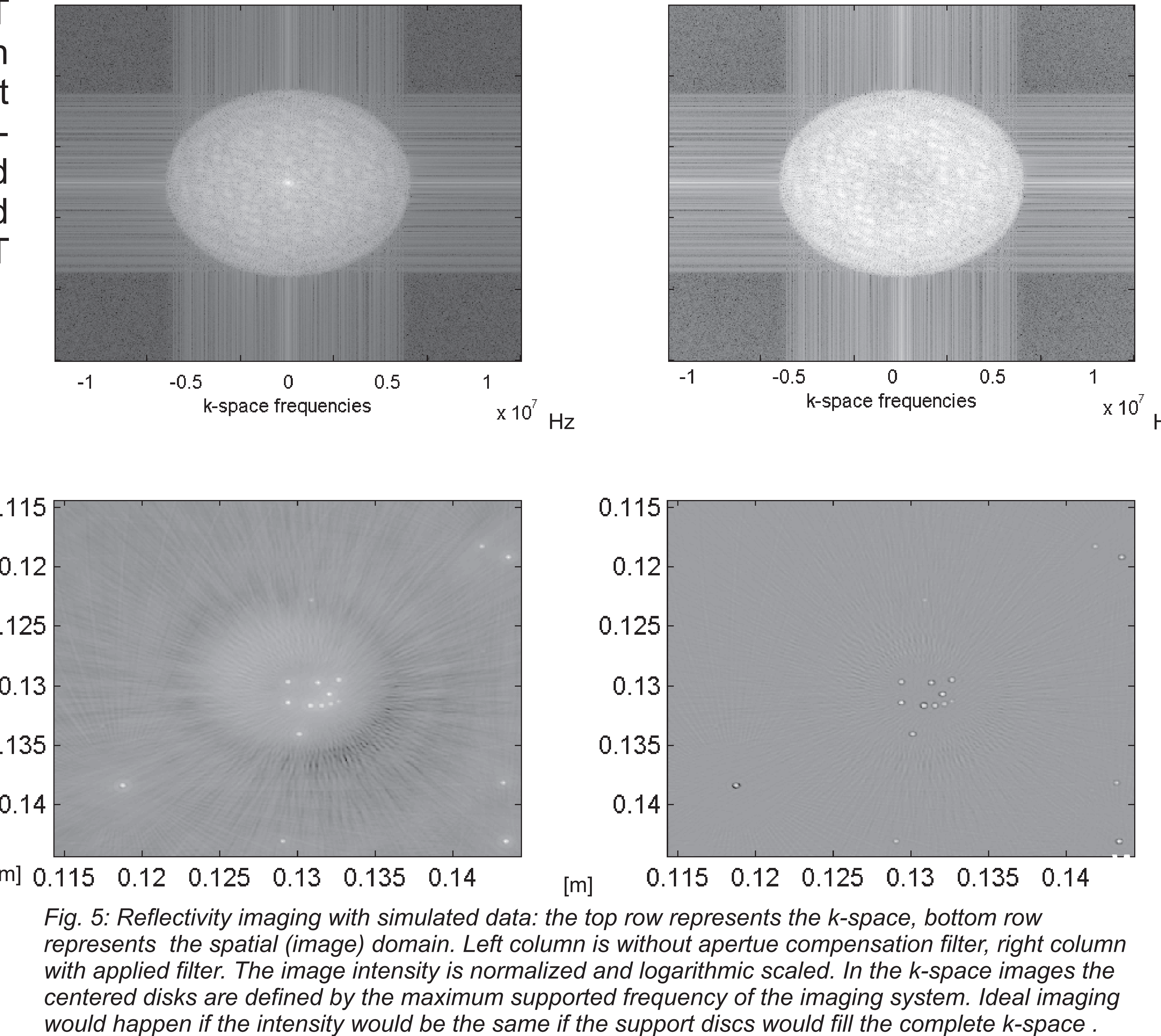

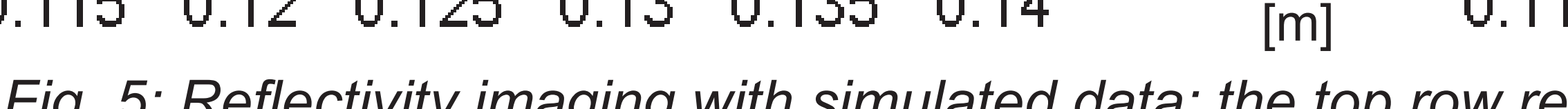

Institute for Data Processing and Electronics Hermann-von-Helmholtz-Platz 76344 Eggenstein-Leopoldshafen Germany

www.ipe.kit.edu

\section{Results}

21 point scatterer simulation \begin{tabular}{l|l|l|l|l}
$\begin{array}{l}\text { Imaging } \\
\text { metrics }\end{array}$ & $\begin{array}{l}\text { Resolved point } \\
\text { scatterers }\end{array}$ & $\begin{array}{l}\text { Mean resolution } \\
\text { in } 10^{-4} \mathrm{~m}\end{array}$ & $\begin{array}{l}\text { Median } \\
\text { resosulution } / \\
\text { in } 10^{-4} \mathrm{~m}\end{array}$ & $\begin{array}{l}\text { Std. deviation } / \\
\text { in } 10^{-4} \mathrm{~m}\end{array}$
\end{tabular}

Straight forward
SAFT imaging

2.52

2.44

2.71

SAFT with aperture
compensation

1.85

1.85

45

\section{Real breast data image}

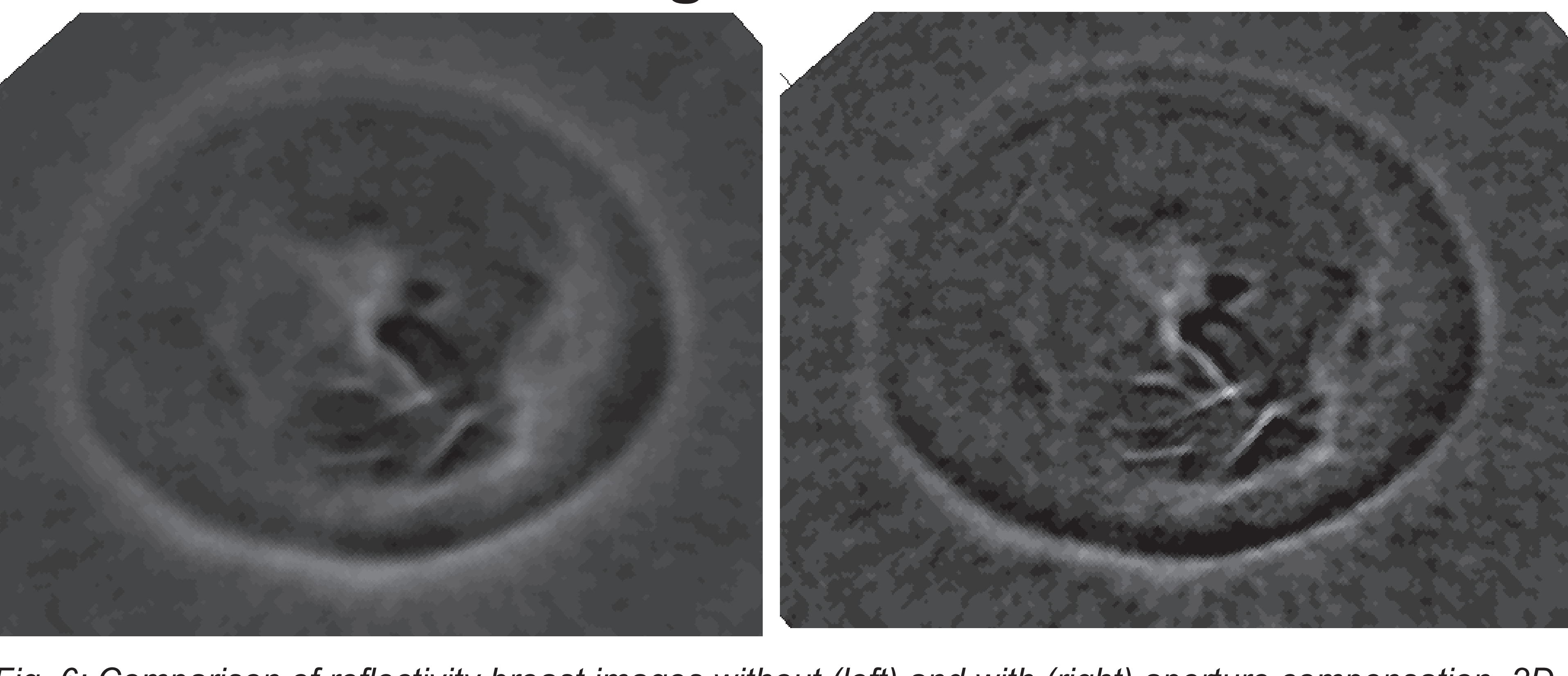

Fig. 6: Comparison of reflectivity breast images without (left) and with (right) aperture compensation. $2 D$

\section{Discussion}

An aperture filter was constructed and the performance regarding the resolution was evaluated. A improvement with simulated point scatterers in the mean resolution over straight-forward SAFT by $26 \%$ was achieved.

Also in images created with real world data a sharpening and increase in resolution could be observed. New tissue structures which were

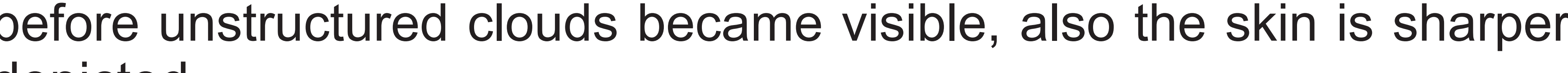
depicted

On the down side, the contrast seems to be slightly degraded as high(requency artifacts, the grating-lobe like SAFT ellipses, are amplified in the imaging, too

REEERENCES

[2] $S$.

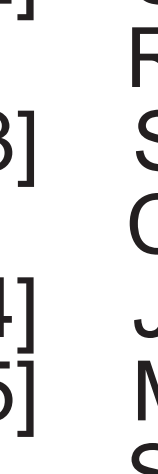

M. Linzer, "Ultrasonic reflectivity imaging in three dimensions

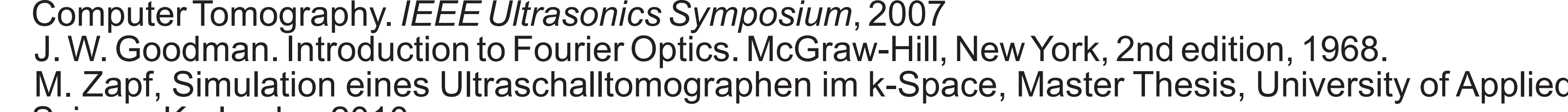

